\title{
Will endoscopic submucosal dissection (ESD) become the gold standard for laterally spreading tumors (LST)?
}

\section{다)(우 $\odot$}

\author{
Authors \\ Henry H.L. Wu ${ }^{1}$, James E. East ${ }^{2}$ \\ Institutions \\ 1 Academic Foundation Programme, Lancaster University \\ and Royal Lancaster Infirmary, University Hospitals of \\ Morecambe Bay, United Kingdom \\ 2 Translational Gastroenterology Unit, Nuffield \\ Department of Medicine, and Oxford NIHR Biomedical \\ Research Centre, Oxford University Hospitals, United \\ Kingdom
}

Bibliography DOI https://doi.org/10.1055/a-0808-4580 | Endoscopy International Open 2019; 07: E260-E263

(C) Georg Thieme Verlag KG Stuttgart · New York ISSN 2364-3722
Corresponding author

Prof. James E. East, Translational Gastroenterology Unit, Experimental Medicine Division, Nuffield Dept of Clinical Medicine, University of Oxford, John Radcliffe Hospital, Headley Way, Headington, Oxford, OX3 9DU, United Kingdom Fax: +44 (0)1865 228763 james.east@ndm.ox.ac.uk
Laterally spreading tumors (LST) have become a recognizable proportion of all pre-cancerous colorectal lesions, particularly with the advent of population-based bowel cancer screening. Since Kudo's first description in 1993 which analyzed how best to remove flat and depressed types of early colorectal cancer via endoscopic mucosal resection (EMR), there has been much debate as to the definition of these lesions and how they should be excised [1]. The 2002 Paris Consensus established boundaries of LST morphology. EMR is currently the mainstream technique for treating LST, but debate has emerged as to whether especially piecemeal EMR should be replaced by newer modalities such as endoscopic submucosal dissection (ESD) [2]. A key point is that en-bloc resection with EMR is usually limited to lesions $<20 \mathrm{~mm}$ diameter in the colon or $<25 \mathrm{~mm}$ in the rectum [3]. Although piecemeal EMR has proven to be a reliable option, this may come with reduced cost-effectiveness over the entire treatment course for the lesion, less good pathological assessment, and increased risks of local recurrence. Because of this, a substantial body of opinion suggests that ESD should be the future in LST treatment. However, when considering ESD as "gold standard," we cannot overlook its downsides of technical intricacy, prolonged time of procedure and increased chances of perforation and overnight hospital admission.

\section{Surgery vs endoscopy}

Major surgical treatment for challenging benign polyps continues to manifest as a popular option due to observed technical limitations with endoscopy in both the United States and Europe. Although focused literature assessing surgical and transanal endoscopic microsurgery (TEM) management in colorectal LST has not been well documented, there is conventional perception amongst surgeons that these remain the most reliable modalities of treatment in achieving curative resection and lowering risks of future recurrence; however, the adverse event (AE) rate very substantially exceeds that of endoscopic therapy for laparoscopic or open resectional surgery with a 30-day mortality at $0.7 \%$ and major postoperative complication rates of $14 \%$ [4]. In this edition of Endoscopy International Open, Russo et al. present a systematic review and meta-analysis comparing endoscopic management options for colorectal LST [5]. LST originally managed via endoscopy required eventual surgery in $2.7 \%$ of cases either due to AEs (pooled rate $0.5 \%$ ), recurrences in follow-up (pooled rate $0.5 \%$ ) or incomplete/ non-curative resection (pooled rate $4.3 \%$ ) [5]. The continued widespread use of major surgery as the popular choice in benign polyp management has recently been questioned in an editorial by Prof. Douglas Rex in Endoscopy, exploring the reluctance of both endoscopists and surgeons to move to endoscopic approaches for both patient and health economic benefits [6]. 


\section{Recurrence risk}

Weighing the balance on determining optimal treatment of LST hinges heavily on rates of recurrence and rates of curative resection if early cancer is found. The meta-analysis from Russo et al. concluded that en-bloc and curative resection rates were significantly higher with ESD compared to EMR. Within subgroup analysis, there has been clear evidence that ESD success in en-bloc resection is not dependent on size of LST while diameter is an important factor to consider for EMR-resected lesions $(53.8 \%,<30 \mathrm{~mm}$ vs $22.3 \%,>30 \mathrm{~mm})$ [5]. In the context of this, it is natural that recurrence rates are significantly more frequent with EMR compared to ESD, particularly for larger lesions $(>30 \mathrm{~mm})$. Such results are supported by Pellise et al., who conducted a multivariate analysis looking at EMR recurrence risk factors [7]. Their study concluded lesion size to be a significant factor: $>40 \mathrm{~mm}$ (OR 8.22, $P<0.001$ ), 31 to $40 \mathrm{~mm}$ (OR $3.44, P=0.002)$ and 21 to $30 \mathrm{~mm}(\mathrm{OR} 2.07, P=0.073)$ compared to $20-\mathrm{mm}$ lesions [7]. Lesion size being a hindering factor for EMR, LST resection does not only apply to en-bloc. Belderbos et al. conducted a meta-analysis illustrating piecemeal EMR recurrence rates for LST $>20 \mathrm{~mm}$ of up to $22 \%$ [8]. This is, therefore, a considerable technical limitation of EMR for larger benign polyps not applicable to ESD and surgery; however, most recurrences are small and can be dealt with effectively by further endoscopic resection [9]. For more challenging recurrence underwater, EMR may be of benefit with Kim et al. demonstrating higher efficacy of underwater vs conventional EMR in the treatment of LST recurrence following previous piecemeal EMR [10].

\section{Risk of early cancer}

Importance of successful en-bloc resection for advanced polyps is summarized in a study by Ronnow et al., showing early invasive cancers ( $\mathrm{T} 1$ ) rate of $12 \%$ for 255 lesions $>2 \mathrm{~cm}$, consistent with other studies showing ranges from $10 \%$ to $23 \%$ [11]. The current suggestion is that for lesion with > Sm 1 , invasion surgery should be performed with resection of local lymph nodes; however, this does not take into account patient wishes or comorbidities [11]. It is of note that for the majority of early lesions up to Sm3 in the Ronnow series, patients preferred endoscopic surveillance [11]. Only 7 of 29 cancers (24\%) underwent surgery with a median follow-up of 13 months. This mirrors the TEM literature where increasingly close surveillance with or without external beam radiotherapy is considered an acceptable option for selected cases. EMR with piecemeal resection has been demonstrated as an independent risk factor for incomplete resection and malignant transformation [5].

\section{Mortality, bleeding and perforation risks}

ESD, EMR and TEM have all shown low acute mortality risks, pooled procedure-related mortality being $0.1 \%$ ( $\triangleright$ Table 1 ) [5]. Though this is surprising considering the portfolio of risks with ESD, Sauer et al. demonstrated no cases of emergency surgery and 30-day mortality for an entire cohort of ESD-treated flat/
Table 1 Comparison of treatment modalities for LST resection.

\begin{tabular}{|l|l|l|l|l|}
\hline & pEMR & ESD & TEM & $\begin{array}{l}\text { Laparoscopic } \\
\text { surgery }\end{array}$ \\
\hline Mortality & $\downarrow$ & $\downarrow$ & $\downarrow$ & $\uparrow$ \\
\hline Morbidity & $\downarrow$ & $\downarrow$ & $\downarrow$ & $\uparrow$ \\
\hline Recurrence rates & $\uparrow$ & $\downarrow$ & Variable & $\downarrow \downarrow$ \\
\hline Cost & $\downarrow$ & $\uparrow$ & $\uparrow \uparrow$ & $\uparrow \uparrow \uparrow$ \\
\hline Hospital stay & $\downarrow$ & $\uparrow$ & $\uparrow$ & $\uparrow \uparrow \uparrow$ \\
\hline
\end{tabular}

LST, laterally spreading tumors; pEMR, piecemeal endoscopic mucosal resection; ESD, endoscopic submucosal dissection; TEM, transanal endoscopic microsurgery

- Table 2 Sydney Endoscopic Recurrence Tool (SERT) [9].

\begin{tabular}{|l|r|}
\hline Risk factor & S \\
\hline LST Size $\geq 40 \mathrm{~mm}$ & 2 \\
\hline Inter-procedural bleeding requiring endoscopic control & 1 \\
\hline High-grade dysplasia & 1 \\
\hline Total & 4 \\
\hline
\end{tabular}

LST, laterally spreading tumor

sessile colorectal neoplasia [12]. No significant differences in delayed bleeding and perforation rate have been shown between the endoscopic treatment modalities but immediate post-procedural bleeding is well-cited to be less frequent with ESD [5].

\section{Health economics}

One approach to examining the significance of achieving enbloc against piecemeal colonic EMR resection in an economic perspective could be analyzed with focus around the four-point Sydney EMR recurrence tool (SERT, > Table 2). This system was described by Tate et al. in 2017 to risk-stratify the likelihood of recurrence of LST after piecemeal EMR [9]. The SERT proposal was that for low-risk lesions (SERT 0, 6-month recurrence rate $9 \%)$ patients could avoid the current recommended guidance of surveillance colonoscopy after 4 to 6 months post-EMR, and simply have a site check at 18 months [9]. Bearing in mind each day-case colonoscopy costs U\$1255 at the Westmead Center, Sydney, this intervention was predicted to save US\$500,000 over an 8-year period [9]. The premise of SERT might be challenged or extended with recent literature demonstrating dramatically lower recurrence rates for all larger-sized LSTs with ESD treatment and R0 margins pathologically with rates of $0.5 \%$ or less ( $\triangleright$ Fig. 1 ) [9]. At this level of recurrence, it might be acceptable to avoid site checks altogether and simply resume surveillance at the appropriate interval for the number and size of polyps, commonly 3 years for lesions $>20 \mathrm{~mm}$ [9]. However, one must acknowledge that the economic benefits of this ESD strategy would come with higher up-front costs, 


\begin{tabular}{|c|c|c|}
\hline SERT vs ESL & & \\
\hline Resection & 6 months & 36 months \\
\hline $\begin{array}{l}\text { SERT 1-4 } \\
\text { Recurrence }\end{array}$ & $23 \% \Rightarrow 36 \%$ & $40 \%$ \\
\hline
\end{tabular}

ESD R0 or

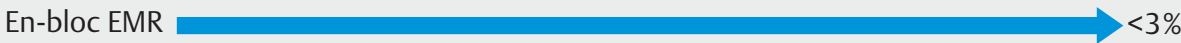

Recurrence

- Fig. 1 Comparision of recurrence rates over time for piecemeal EMR according to SERT status verus en bloc EMR or ESD with clear margins (RO).

but perhaps lower costs overall when the entire cycle from resection and site checks to confirmation of lesion eradication at 36 months is taken into account ( $\downarrow$ Table 1, $>$ Fig. 1). Longer procedure time, equipment costs, complications and higher chances of overnight hospital stay with ESD are well-documented, particularly for challenging LSTs.

\section{East versus west}

Another area of consistent discussion is the discrepancy in ESD performance outcomes between European and Asian studies. ESD is generally considered as one of the more difficult techniques in endoscopy. There is a steep learning curve and operator expertise plays an important role in its success. Emphasizing its benefits, Japan has pioneered widespread use of ESD for many gastrointestinal lesions in addition to LST since the early $1990 \mathrm{~s}$ and endoscopists received focused sessions on ESD technique from early subspecialist training [13]. ESD services are provided in both tertiary and district-level settings. Recognizing that not all colorectal lesions are suited to ESD, there has always been careful patient selection before ESD is performed to ensure high likelihood of R0 resection and histopathologyconfirmed clear margins. This explains the excellent en-bloc results (many studies showing up to $90 \%$ ) that have emerged from Japanese centers over the years, although recognizable complication rates would be inevitable due to risks associated with ESD [13]. Constrained by resource provision and foreseeing the potential strain of post-procedural complications and prolonged hospital stay on the already pressured workforce and bed spaces, western centers have traditionally opted for EMR as the safer treatment modality across high-volume hospitals [13]. Due to limitations of EMR in LST treatment, ESD alongside other newer full-thickness resection technologies has been more popular with western investigators attempting to identify ways to improve long-term patient outcomes [13]. Lack of operator experience and a rigid patient selection pathway still in development has meant that ESD has yet to provide convincing evidence in the west for being the gold-standard modality of LST management.

\section{Conclusion}

On balance, it is sensible to suggest that ESD should be considered gold standard for higher-risk LSTs (LST-NG, LST-G with large nodule, and larger lesions $>40 \mathrm{~mm}$ ) where rates of recurrence are substantial and risks of problems with pathological interpretation are higher without clean, complete en-bloc resection. En-bloc EMR would ideally be the optimal choice for smaller lower-risk granular-type LSTs with acceptable outcomes, and avoidance of inter-procedural and post-procedural risks frequently associated with ESD. Comorbidities, and patient choice should factor into the decision-making process for LSTs that do not fall into either category convincingly. Progressive use of ESD in the west will generate further data to illustrate its validity and reliability for LST excision. With the aim of reducing disadvantages, we hope the advent of new endoscopic resection platforms, lifting agents, and resection devices will gradually swing the pendulum towards conventional use of en bloc resection for all LSTs in the future.

\section{Acknowledgements}

Dr East received funding from the National Institute for Health Research (NIHR) Oxford Biomedical Research Centre (BRC). The views expressed are those of the author(s) and not necessarily those of the NHS, the NIHR or the Department of Health.

\section{Competing interests}

Prof. James East has served on clincal advisory boards for Lumendi and Boston Scientific.

References

[1] Kudo S. Endoscopic mucosal resection of flat and depressed types of early colorectal cancer. Endoscopy 1993; 25: 455-461

[2] The Paris endoscopic classification of superficial neoplastic lesions: esophagus, stomach, and colon: November 30 to December 1, 2002. Gastrointest Endosc 2003; 58: S3-43 
[3] Ferlitsch M, Moss A, Hassan C et al. Colorectal polypectomy and endoscopic mucosal resection (EMR): European Society of Gastrointestinal Endoscopy (ESGE) clinical guideline. Endoscopy 2017; 49: $270-297$

[4] Peery AF, Cools KS, Strassle PD et al. Increasing rates of surgery for patients with nonmalignant colorectal polyps in the United States. Gastroenterology 2018; 154: 1352 - 1360

[5] Russo P, Barbeiro S, Libanio D et al. Management of colorectal laterally spreading tumours: a systematic review and meta-analysis. Endosc Int Open 2019; 07: E239-E259

[6] Rex DK. If endoscopic mucosal resection is so great for large benign colon polyps, why is so much surgery still being done? Endoscopy 2018; 50: 657-659

[7] Pellise M, Burgess NG, Tutticci N et al. Endoscopic mucosal resection for large serrated lesions in comparison with adenomas: a prospective multicentre study of 2000 lesions. Gut 2017; 66: 644-653
[8] Belderbos TD, Leenders M, Moons LM et al. Local recurrence after endoscopic mucosal resection of nonpedunculated colorectal lesions: systematic review and meta-analysis. Endoscopy 2014; 46: 388-402

[9] Tate DJ, Desomer L, Klein A et al. Adenoma recurrence after piecemeal colonic EMR is predictable: the Sydney EMR recurrence tool. Gastrointestinal Endoscopy 2017; 85: 647-656

[10] Kim HG, Thosani N, Banerjee S et al. Underwater endoscopic mucosal resection for recurrences after previous piecemeal resection of colorectal polyps (with video). Gastrointest Endosc 2014; 80: 1094- 1102

[11] Rönnow CF, Elebro J, Toth E et al. Endoscopic submucosal dissection of malignant non-pedunculated colorectal lesions. Endosc Int Open 2018; 6: E961

[12] Sauer M, Hildenbrand R, Oyama T et al. Endoscopic submucosal dissection for flat or sessile colorectal neoplasia $>20 \mathrm{~mm}$ : A European single-center series of 182 cases. Endosc Int Open 2016; 4: E895

[13] Burgess NG, Bourke MJ. Endoscopic resection of colorectal lesions: The narrowing divide between East and West. Digest Endosc 2016; 28: $296-305$ 